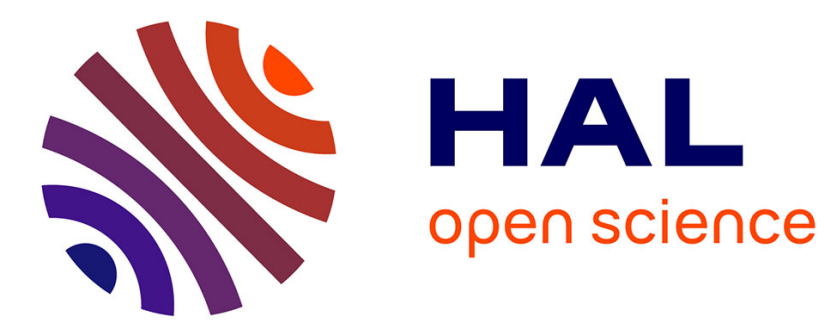

\title{
Réussir des réformes en France
}

Pierre Cahuc, André Zylberberg

\section{To cite this version:}

Pierre Cahuc, André Zylberberg. Réussir des réformes en France. Commentaire, 2009, 32 (128), pp.955-965. halshs-00638085

\section{HAL Id: halshs-00638085 \\ https://shs.hal.science/halshs-00638085}

Submitted on 3 Nov 2011

HAL is a multi-disciplinary open access archive for the deposit and dissemination of scientific research documents, whether they are published or not. The documents may come from teaching and research institutions in France or abroad, or from public or private research centers.
L'archive ouverte pluridisciplinaire HAL, est destinée au dépôt et à la diffusion de documents scientifiques de niveau recherche, publiés ou non, émanant des établissements d'enseignement et de recherche français ou étrangers, des laboratoires publics ou privés. 


\section{Comment réussir des réformes en France?}

Pierre Cahuc, André Zylberberg

Mai 2009

Le 6 Mai 2007, Nicolas Sarkozy est élu Président de la République. Son programme promet le changement à une France réputée irréformable et championne de l'immobilisme. Comment peut-il réussir là où tous les autres ont échoué ?

Des échecs retentissants, la France en a connus beaucoup, y compris dans un passé proche. En 1984, après plusieurs années passées à batailler, François Mitterrand abandonne son grand projet de service public unifié et laïque de l'éducation nationale. En 1993, Edouard Balladur échoue à imposer le «Smic jeune » qui propulse dans la rue des centaines de milliers de manifestants. En 1995, Alain Juppé veut aligner le système de retraites du secteur public sur celui du privé. Plusieurs semaines de grèves qui paralysent le pays l'obligent à retirer son projet. Deux ans plus tard, la droite le paye cher: elle perd les élections législatives et Jacques Chirac doit cohabiter pendant 5 ans avec un gouvernement dirigé par Lionel Jospin. Quelques années plus tard, malgré ces expériences traumatisantes, Dominique de Villepin croit pouvoir faire passer aux forceps le Contrat Première Embauche (CPE) qui prévoit une absence de protection contre le licenciement pendant deux ans pour les jeunes de moins de 25 ans. Le même scénario de manifestations à répétition l'accule à une piteuse capitulation et met fin à ses ambitions présidentielles.

\section{La stratégie de Nicolas Sarkozy}

Tous ces événements, Nicolas Sarkozy les a souvent vécus aux premières loges. Il en a tiré une conviction : les Français veulent des réformes, mais tout passage en force est voué à l'échec. Quitte à en décevoir quelques uns, il ne sera pas le clone français de Margaret Thatcher. Le titre même de son projet présidentiel Ensemble, tout devient possible qu'ont pu lire 45 millions d'électeurs témoigne de sa volonté de se démarquer de ses prédécesseurs. La critique du passé est clairement affichée lorsqu'il affirme "je vous associerai au choix des réformes. Je crois que l'on prend de meilleures décisions si l'on prend le temps d'écouter ceux qui sont concernés sur le terrain, et que les réformes sont mieux 
appliquées si chacun a pu au préalable les comprendre et les accepter ». Dans le même texte, il annonce « je m'appuierai fortement sur le dialogue social, qui est un des grands manques de notre payss. Avant même sa prise de fonction officielle, Nicolas Sarkozy reçoit au pavillon de la Lanterne, accolades à l'appui, les représentants de toutes les organisations syndicales. En réalité, la méthode de Nicolas Sarkozy repose sur deux principes : l'étouffement et la conciliation.

\section{L'étouffement}

En ouvrant constamment de nombreux chantiers Nicolas Sarkozy cherche à étouffer tous ses opposants réels ou supposés. Les parlementaires doivent voter toutes les lois importantes relevant du domaine économique et social en respectant la procédure d'urgence qui limite les allées et venues entre les deux assemblées. Quant aux partenaires sociaux, ils sont sommés de négocier puis de proposer des projets de réformes dans des délais très brefs. Ce principe permet à Nicolas Sarkozy de maîtriser l'agenda des réformes, de limiter les situations de blocage généralisé et, surtout, d'éviter qu'une concession sur une mesure soit perçue comme un échec pour l'ensemble. L'annonce de l'Elysée sur la réforme de l'Etat comprend 166 mesures et le rapport de la Commission pour libérer la croissance française, présidée par Jacques Attali ${ }^{1}$, ne contient pas moins de 316 décisions $^{2}$. Si quelques unes sont abandonnées en cours de route, il n’y aura pas grand dommage ${ }^{3}$.

\section{La conciliation}

Lorsqu'elles s'expriment avec force, les revendications catégorielles doivent être satisfaites. C'est parce que les bénéfices attendus de l'ensemble des chantiers ouverts sont très importants qu'on peut penser qu'il y a peu à perdre en cédant sur quelques revendications catégorielles. En effet, ne pas céder risquerait de déclencher des conflits susceptibles de compromettre l'ensemble des projets. Les partenaires sociaux sont particulièrement avantagés, puisque Nicolas Sarkozy s'est engagé à transformer en projet de loi tout accord interprofessionnel concernant la modernisation du marché du travail, la représentativité syndicale et l'assurance

\footnotetext{
${ }^{2}$ Jacques Attali, Rapport de la Commission pour la libération de la croissance française : 300 décisions pour changer la France, La Documentation Française, 2008. Nicolas Sarkozy et François Fillon ont demandé à Jacques Attali de rendre un rapport pour « examiner les conditions d'une libération de la croissance française » pour la fin du mois de décembre 2007 dans une lettre de mission datée du $1^{\text {er }}$ août 2007. Cette lettre peut être consultée à l'adresse : www.liberationdelacroissance.fr/files/rapports/lettredemission.pdf.

${ }^{3}$ Elie Cohen qualifie cette stratégie de «carpet bombing» des réformes. L'objectif étant de provoquer un état de désorientation et même de sidération de l'adversaire. Voir son article «Les trois erreurs de Nicolas Sarkozy » publié le 9 mai 2008 sur le site Telos, disponible à l'adresse www.telos-eu.com/fr/article/sarkozy le reformateur.
} 
chômage. Les partenaires sociaux sont ainsi invités à «s'auto-réformer». Pour d'autres, c'est moins simple: il faut se mobiliser pour se faire entendre. En la matière, c'est généralement Nicolas Sarkozy en personne qui tranche... et qui communique.

\section{2.. La stratégie en pratique}

Voilà pour la stratégie. Mais l'histoire nous apprend que les réformes n'atteignent pratiquement jamais la totalité de leurs objectifs, loin de là. Comment juger alors de la réussite d'une réforme ? Bien sûr en comparant la situation initiale à celle qui prévaut après la réforme. Mais une réforme comporte de multiples dimensions. Un texte de loi ou un protocole d'accord contient souvent des dizaines d'articles, d'alinéas et de sous alinéas. La mise en œuvre des décisions et les réactions des protagonistes ont aussi leur importance. Il est bien connu que le diable se loge toujours dans les détails. Porter un jugement circonstancié sur la réussite ou l'échec d'une réforme nécessite d'avoir une vision claire des conséquences de tous ces éléments pris dans leur ensemble. C'est un exercice particulièrement délicat et souvent rébarbatif. La réforme des régimes spéciaux de retraites de la SNCF, de la RATP, d'EDF et de GDF, menée au pas de charge à l'automne 2007, en est une très bonne illustration ${ }^{4}$.

\section{La réforme des régimes spéciaux de retraite}

La réforme des régimes spéciaux est la mère des batailles pour Nicolas Sarkozy : s'il renonce, sa crédibilité est anéantie et il se «chiraquise » pour le restant de son quinquennat. A l'inverse, s'il gagne cette bataille, il prouve que sa méthode est la bonne, puisqu'il aura réussi là où ses prédécesseurs ont échoué, ou n’ont même pas osé essayer. L’enjeu de la réforme, qui a le soutient d'une très large majorité de Français, est rappelé le 11 octobre 2007 sur le site officiel du Premier Ministre ${ }^{5}$ : L'objectif est de réformer ces régimes en harmonisant, pour le présent et pour l'avenir, leurs principaux paramètres avec ceux applicables au régime de retraite de la fonction publiques. La formulation du Premier Ministre laisse déjà planer un doute. S'il est question d'harmoniser les «principaux» paramètres des régimes de retraite, est-ce que cela signifie que d'autres paramètres considérés « secondaires » ne seront pas harmonisés ? D’ailleurs, à bien y réfléchir, comment fait-on pour distinguer un paramètre principal d'un paramètre secondaire? En pratique, seul importe l'effet global de tous les paramètres sur l'équilibre d'ensemble du projet. En matière de retraite, le niveau

\footnotetext{
${ }^{4}$ Notre propos n'est pas ici de porter un jugement sur le caractère souhaitable ou non de la réforme des régimes spéciaux, mais de mettre en évidence l'écart entre les objectifs des réformes de Nicolas Sarkozy et leur réalisation.

${ }^{5} \mathrm{http}: / /$ www.premier-ministre.gouv.fr/chantiers/retraites_867/reforme_regimes_speciaux_1220/
} 
des pensions versées et donc le coût pour la collectivité dépendent d'une multitude de paramètres, citons par exemple : la durée de cotisation, les variables qui servent à indexer le niveau des pensions (inflation ou salaire moyen), le degré d'indexation des pensions, le taux de remplacement, c'est-à-dire le rapport entre la pension et les rémunérations perçues pendant la vie active, la chronologie des salaires et des diverses primes retenues pour calculer la pension, les possibilités de rachat de points de retraite pour les années d'étude, la prise en compte des congés formation, etc.

A la lumière des informations (parcellaires) sur les accords finaux signés entre les directions des entreprises et les représentants de leur personnel, on constate effectivement que certains changements permettront de faire des économies. Il en va ainsi de la durée de cotisation des agents de la SNCF, de la RATP, d'EDF et de GDF qui sera progressivement alignée sur celle du privé et de la fonction publique. Mais, par ailleurs, et vraisemblablement en contrepartie, la réforme permet d'intégrer des primes dans les rémunérations qui servent de base au calcul des retraites. C'est un puissant levier pour accroittre significativement les pensions, calculées en fonction des six derniers mois de salaire et non des vingt cinq meilleures années de salaire comme pour les salariés du privé. En outre, la réforme a prévu l'ouverture de négociations sur la pénibilité au travail afin de permettre aux personnes dont les métiers sont difficiles de pouvoir partir plus tôt à la retraite. Comme s'en inquiète Dominique Leclerc, le rapporteur au Sénat sur le projet de loi de financement de la sécurité sociale pour 2008, des négociations à rebondissement risquent de conduire à la création d'une nouvelle forme déguisée de préretraite ${ }^{6}$. Bref, comme tous les paramètres bougent ou sont susceptibles de bouger, il est très difficile de savoir si la réforme des régimes spéciaux pour le personnel actuellement en poste permet de réaliser vraiment des économies par rapport à une situation où l'on aurait maintenu tels quels les régimes de retraite de ces personnels.

On aurait pu s'attendre à ce que les techniciens de Bercy évaluent précisément l'impact de la réforme sur les finances publiques et que cette information soit publique. Il n'en est rien. Nous avons cherché à obtenir cette information auprès des services compétents et nous avons eu l'impression de nous heurter à un véritable secret d'Etat si bien que nous doutons, au moment où nous écrivons ces lignes, que cette évaluation existe. Nous ne sommes d'ailleurs pas les seuls à aboutir à cette conclusion. En juillet 2008, le rapport du Sénat sur la caisse de retraite de la RATP "fait part de son étonnement, alors que la réforme est engagée, sur le fait que le gouvernement déclare qu'il n'a jamais fixé de cadrage financier à la réforme des régimes spéciaux». La Direction du budget a affirmé au

\footnotetext{
${ }^{6}$ Dominique Leclerc, Rapport au nom de la commission des Affaires sociales du Sénat sur le projet de loi de financement de la sécurité sociale pour 2008, Tome IV : Assurance vieillesse, page 58.
} 
Sénateur Bertrand Auban, vice président du groupe socialiste et rapporteur au Sénat sur ce dossier, "ne pas pouvoir déterminer avec précision le nivean des économies ». En toute logique, le rapport estime qu'il est «permis de s'interroger sur la portée financière de la réforme des régimes spéciaux à moyen terme », et notamment de se demander si la subvention d'équilibre de l'Etat sera inférieure ou non «à ce qu'elle aurait été sans la réforme» ${ }^{7}$. En clair, le rapporteur du Sénat s'avoue incapable de savoir si la réforme des régimes spéciaux va coûter moins cher à la collectivité comparée à une situation où l'on n'aurait pas touché au régime de retraite de ces personnels !

\section{La difficile évaluation des réformes économiques et sociales des dix-buit premiers mois du quinquennat}

L'épisode de la réforme des régimes spéciaux montre à quel point il est difficile de juger le résultat final. Comme tous les paramètres ont bougé en même temps, il faudrait avoir plus d'informations que celles que nous possédons (et visiblement les parlementaires ne les possèdent pas non plus) pour porter une appréciation raisonnée. Comment le gouvernement a-t-il pu avoir l'objectif de faire des économies sans s'appuyer sur des évaluations chiffrées, alors que tous les spécialistes savent que c'est indispensable? Les services de Bercy et des divers ministères regorgent de techniciens chevronnés qui ont alerté les hommes politiques de la nécessité de mener à bien ces évaluations. En réalité, il semble que le coût de la réforme importe peu. L'essentiel est de produire l'apparence du changement. Pour cela, il suffit de brandir dans la multitude des modifications celles qui accréditent le mieux cette idée. L’alignement de la durée de cotisation sur celle du régime général pour les nouveaux entrants en fait évidemment partie. Dans le même temps, le gouvernement peut (discrètement) accorder de substantielles compensations aux actuels salariés des régimes spéciaux en jouant sur d'autres paramètres, comme les primes ou l'octroi de fortes augmentations de salaires en fin de carrière. Tout le monde est satisfait: l'opinion publique, qui a l'impression de vivre désormais dans un système moins inégalitaire, et les bénéficiaires des régimes spéciaux qui n’ont rien perdu (et peut être même gagné un peu). On comprend mieux, dans ces circonstances, pourquoi la réforme a pu passer après seulement neuf jours de grève. Le contribuable est vraisemblablement le seul perdant, mais pour l'instant il l'ignore. L'aggravation des déficits est un poison à diffusion lente. Mais à court terme c'est peu cher payé pour une aussi belle réussite politique. Ce dossier étant réglé, on peut continuer à étouffer les adversaires et l'opinion publique en passant aux réformes suivantes, qui sont déjà d'ailleurs en route. Cette stratégie fait émerger l'idée que Nicolas Sarkozy n’a peut être pas

\footnotetext{
${ }^{7}$ Bertrand Auban, Rapport d'information au nom de la commission des Finances, du contrôle budgétaire et des comptes économiques de la Nation du Sénat sur la caisse de retraite de la RATP, page 27, Annexe au procès verbal de la séance du 9 juillet 2008 .
} 
réformé autant qu’il le prétend, mais que des changements, plus ou moins notables selon les analystes, doivent être portés à son actif. Comme l'explique avec sérénité Raymond Soubie, conseiller social de Nicolas Sarkozy, "mieux vaut faire une réforme à $80 \%$ qui passe, qu'une réforme à $100 \%$ rejetée par les partenaires sociaux $\rangle^{8}$.

La stratégie adoptée par Nicolas Sarkozy ne serait donc finalement pas si mauvaise dans un pays réputé irréformable. Dans notre ouvrage, nous contestons radicalement ce jugement. Les doutes qui se sont manifestés à l'occasion de la réforme des régimes spéciaux nous ont conduits à explorer les réformes économiques et sociales menées entre mai 2007 et janvier 2009. Elles ont des objectifs ambitieux : diminuer le chômage, «réhabiliter» le travail et accroître le pouvoir d'achat. Elles sont nombreuses : sur cette période, des lois ont été votées, des accords ou des protocoles ont été signés pour changer le contrat de travail, les possibilités d'implantation des grandes surfaces, la situation des professions réglementées (en l'occurrence les taxis), la représentativité des organisations syndicales, les fondements de la lutte contre la pauvreté et la réglementation du temps de travail. Contrairement à ce que nous avons regretté à propos des régimes spéciaux, nous disposons de suffisamment d'informations sur ces réformes pour porter un jugement fiable. En suivant le cheminement souvent tortueux qui mène des intentions initiales aux résultats, une conclusion s'impose: les réformes réalisées pendant les dix-huit premiers mois de la présidence de Nicolas Sarkozy n’ont aucune raison de réduire le chômage, d'accroittre l'emploi ou d'améliorer le pouvoir d'achat. Elles ont même plutôt tendance à aggraver les problèmes qu'elles devaient résoudre tout en creusant le déficit public.

A y regarder de plus près, l'échec des réformes réalisées dans les 18 premiers mois du quinquennat de Nicolas Sarkozy est la conséquence de la stratégie adoptée. Celle-ci repose sur le pari qu'on peut réformer en étouffant l'adversaire tout en étant conciliant lorsque son pouvoir de résistance est élevé. En pratique, l'étouffement n'a pas eu l'effet escompté. Dans tous les domaines le gouvernement a pensé prendre de vitesse les groupes dont les intérêts étaient menacés par la réforme en imposant des délais de concertation très courts. Mais ces groupes sont très bien organisés. Ils se mobilisent rapidement. Leurs capacités d'expertise sont souvent supérieures à celle des cabinets ministériels et des parlementaires. Ils savent s’adapter aux exigences de changement de quelques «paramètres » emblématiques réclamés par le gouvernement tout en s'arrangeant pour en modifier d'autres à leur avantage, et sans publicité. Finalement, ils sortent

\footnotetext{
${ }^{8}$ «Portrait : Raymond Soubie, conseiller social de Nicolas Sarkozy », Challenges, $\mathrm{n}^{\circ} 142$, du 30 octobre au 5 novembre 2008 , page 80 .
} 
gagnants des réformes ${ }^{9}$. Pour le constater, il faut lire avec minutie des textes de lois ou d'accords effroyablement compliqués qui ont fleuri au cours des 18 premiers mois du quinquennat. Nicolas Sarkozy et son gouvernement ont été pris à leur propre piège. Ils se sont trouvés dans la position de l'étouffeur étouffé, obligés d'endosser des lois ou des accords de la catégorie « usine à gaz » qu'ils n'avaient plus le temps de modifier (dans l'hypothèse où ils l'auraient voulu) à cause des délais qu'ils avaient eux-mêmes imposés. Nicolas Sarkozy s'est donc pris à son propre piège. Cet échec est avant tout celui d'une méthode.

\section{Les racines de l'échec des réformes}

Pour sonder les causes profondes de l'échec des réformes, il est instructif d'observer ce qui se passe à l'étranger.

\section{La qualité de la démocratie}

De nombreuses réformes sont impulsées par les programmes d'aides de la Banque Mondiale ou du Fonds Monétaire International. Ces organisations concèdent des prêts à condition que les pays emprunteurs réalisent des réformes supposées favorables à leur développement. Ces programmes, dont le bien-fondé est débattu, définissent généralement des objectifs clairs, comme une diminution de l'inflation ou des déficits publics. Dans ces conditions, il est assez facile de savoir si une réforme a atteint ses objectifs. Or, la Banque Mondiale a constaté qu'environ un tiers échouent. David Dollar et Jakob Svensson ${ }^{10}$ ont identifié l'origine de ces échecs en examinant plus de deux cents réformes entre 1980 et 1995. Leur principal résultat peut surprendre : ils trouvent que la réussite des réformes repose sur la qualité des institutions du pays qui les met en œuvre et non sur les moyens octroyés par la Banque Mondiale. Ils constatent même que celle-ci consacre beaucoup plus de ressources aux programmes qui se soldent par un échec! David Dollar et Jakob Svensson montrent, par exemple, que les pays dont le

\footnotetext{
${ }^{9}$ Le constat que les réformes peuvent avoir des résultats opposés à ceux escomptés n'est pas nouveau comme nous le verrons plus en détail dans la conclusion de cet ouvrage. Plusieurs contributions ont étudié les conséquences des programmes d'aides de la Banque Mondiale, qui s'accompagnent de réformes structurelles. L'étude de David Dollar et Jakob Svensson, "What Explains the Success or Failure of Structural Adjustment Programmes?" Economic Journal, vol 110, pp. 894-917, 2000, montre que ces programmes réussissent mieux dans les pays plus démocratiques. Daron Acemoglu, Simon Johnson, Pablo Querubin et James A. Robinson "When Does Policy Reform Work - The Case of Central Bank Independence", 2008, à paraittre dans les Brookings Papers on Economic Activity, aboutissent à la même conclusion pour les réformes des banques centrales.

10 "What explains the success or failure of structural adjustment programs ?", The Economic Journal, 110 (466), octobre 2000, pp. 894-917.
} 
gouvernement n'est pas élu démocratiquement, ou dont le chef d'Etat est au pouvoir depuis longtemps, échouent bien plus souvent que les autres quels que soient les moyens alloués par la Banque Mondiale. Corruption et absence de transparence rendent impossible la maitrise des dépenses publiques et l'instauration d'une réelle indépendance des autorités monétaires.

Une autre étude va encore plus loin dans le diagnostic. Elle montre que les réformes de la législation ne sont pas suffisantes et qu'elles peuvent même accentuer les dysfonctionnements de l'économie si des groupes puissants continuent à exploiter les privilèges dont ils jouissaient avant les réformes ${ }^{11}$. L'expérience des pays de l'Est après la chute du mur de Berlin en 1989 illustre bien ce constat. Avant cet événement, la plupart de ces pays vivaient sous un régime d'économie planifiée où un organisme central fixait le prix de chaque produit et la production correspondante. Ce système a donné maintes fois la preuve de son inefficacité. La libéralisation des prix s'imposait comme une des principales réformes des économies post-socialistes. Mais cette libéralisation n'a pas touché tous les secteurs en même temps. Ainsi, des entreprises publiques ont continué à vendre des matières premières selon des prix administrés très bas tandis que les prix des biens finaux qui utilisaient ces matières premières étaient libérés. Les membres bien placés de l'ancien régime ont pu alors s'approvisionner en matières premières bon marché auprès des entreprises publiques pour produire des biens finaux dont ils avaient le monopole ${ }^{12}$. Ils engrangeaient ainsi des profits exceptionnels et faisaient ensuite pression pour que les prix des matières premières restent régulés et que leur monopole sur les biens finaux soit conservé. La réforme du système de fixation des prix, sous la forme d'une libéralisation partielle, a conduit à une situation pire que celle qui existait lorsque tous les prix étaient administrés. Une petite minorité a pu rapidement amasser une fortune colossale sur le dos du reste de la population. La richesse pharamineuse de cette minorité lui a permis de corrompre les gouvernements pour pérenniser et étendre ses privilèges. Pourtant la privatisation des entreprises et la fin du régime des prix administrés étaient des réformes indispensables dès lors que les pays de l'ex bloc soviétique aspiraient à commercer avec le reste du monde. Des réformes qui auraient pu améliorer substantiellement le fonctionnement de l'économie ont eu des effets opposés ${ }^{13}$. De toutes ces expériences, une leçon se dégage : la conduite des réformes conditionne totalement le résultat final. Et la conduite des réformes dépend en grande partie de la qualité des institutions du pays.

\footnotetext{
${ }^{11}$ Daron Acemoglu, Simon Johnson, Pablo Querubin et James Robinson, « When Does Policy Reform Work - The Case of Central Bank Independence", à paraître dans les Brookings Papers on Economic Activity.

${ }^{12}$ Voir l'article de Joel Hellman, "Winners take all: The Politics of Partial Reform in Postcommunist Transitions", World Politics Vol. 50, 1998, pp. 203-234.

${ }^{13}$ Sur ces points, voir l'analyse de Anders Olofsgård, "The Political Economy of Reform: Institutional Change as a Tool for Political Credibility”, Working Paper n³1358, World Bank.
} 
Apparemment toutes ces expériences exotiques n’ont rien à voir avec la France, pays développé fier de son modèle social et de ses institutions républicaines. Pas si sûr. La Banque Mondiale et d'autres organisations non gouvernementales publient régulièrement des indicateurs de la qualité de la gouvernance d'un pays. Ces indicateurs portent principalement sur l'efficacité du gouvernement et de l'administration, la qualité de la réglementation, le respect du droit ou encore le degré de corruption ${ }^{14}$. Pour tous ces indicateurs, la France est systématiquement mal classée au sein des pays développés ${ }^{15}$. Ainsi, pour la corruption et l'efficacité du gouvernement et de l'administration, la France arrive en $23^{\text {ème }}$ position, loin derrière les pays scandinaves, la Grande Bretagne ou l'Allemagne. Les Etats-Unis sont devant la France, même pour l'efficacité du gouvernement et de l'administration. Quant à la qualité de la réglementation, la France est en $28^{\text {ème }}$ position, juste derrière Chypre et Malte. En moyenne, pour l'ensemble des indicateurs disponibles, la France se classe $21^{\mathrm{e} m e}$. Le Danemark arrive en tête, suivi par la Suisse, la Finlande, l'Islande et la Suède. Or, les bonnes performances économiques de ces pays, qui concilient un niveau d'emploi et un revenu par habitant élevés avec de faibles inégalités, sont profondément enracinées dans l'efficacité de leur gouvernement, de leur administration et de leur réglementation. C'est parce que leurs citoyens ont confiance dans un Etat providence plus transparent et plus efficace qu'ailleurs qu'ils peuvent mettre en œuvre des réformes et s'adapter au changement ${ }^{16}$. Essayer de réformer tout azimuts dans un environnement qui ne possède pas ces qualités a toutes les chances d'échouer.

L'examen des multiples expériences menées depuis des décennies dans de nombreuses régions du monde fournit une conclusion claire : le manque de transparence, la faible crédibilité du gouvernement et de l'administration ainsi que la corruption sont les pires ennemis de la réforme. Or, c'est essentiellement le manque de transparence et le manque de crédibilité dans la

\footnotetext{
${ }^{14}$ Plus précisément, six dimensions de la qualité de la gouvernance sont prises en compte:

$1^{\circ}$ ) les moyens d'expression des citoyens et le degré de responsabilité des dirigeants ;

$2^{\circ}$ ) la stabilité politique et l'absence de violence, qui mesure la perception de la probabilité que le gouvernement puisse être déstabilisé ou renversé par des moyens anticonstitutionnels ou violents.

$3^{\circ}$ ) l'efficacité du gouvernement et de l'administration, qui mesure la perception de la crédibilité des engagements du gouvernement et de la qualité des services de l'administration.

$4^{\circ}$ ) la qualité de la réglementation, qui mesure la perception de la capacité du gouvernement de formuler et d'appliquer des réglementations qui promeuvent le développement du secteur privé.

$5^{\circ}$ ) la règle de droit, qui mesure la confiance dans la qualité du droit, et en particulier la qualité de l'exécution des contrats, les droits de propriété, la police et les tribunaux, ainsi que les risques de criminalité et de violence.

$6^{\circ}$ ) la corruption qui mesure la perception de la mesure dans laquelle le pouvoir des agents de l'Etat est exercé des fins lucratives, y compris les petits et les grands formes de la corruption, ainsi que de "capture" de l'État par les élites et les intérêts privés

${ }^{15}$ L'étude la plus complète est celle de la Banque Mondiale. Elle est décrite dans l'article de Daniel Kaufmann, Aart Kraay et Massimo Mastruzzi, «Governance Matters VII: Aggregate and Individual Governance Indicators 19962007”, World Bank Policy Research Department Working Paper, 2008, disponible à l'adresse www.worldbank.org/wbi/governance/govdata.

${ }^{16}$ Voir Yann Algan et Pierre Cahuc, 2007, La société de défiance : comment le modèle social français s'auto-détruit? Edition rue d'Ulm.
} 
maitrise du processus des réformes qui sont à l'origine des échecs de Nicolas Sarkozy. Le ton était donné dès l'épisode de la réforme des régimes spéciaux de retraite en novembre 2007, six mois après le début du quinquennat. L’objectif affiché était de diminuer les coûts exorbitants de ces régimes en allongeant les durées de cotisations. Face à la résistance des personnes concernées, le gouvernement a négocié sous le manteau, en concédant des avantages contre un allongement de la durée de cotisation, et en se gardant bien de publier un seul document permettant d'évaluer les économies réalisées grâce à cette réforme. Le "succès» a pu être affiché, mais il n'était qu'apparent et, finalement, que de courte durée. Tous ceux dont les intérêts pouvaient être menacées par les projets annoncés de Nicolas Sarkozy ont vite compris que le gouvernement était prêt à concéder beaucoup pour que les réformes se «réalisent». Ils savaient dès lors que des arrangements leur permettant même d'améliorer leurs situations étaient possibles. Le lobbying, les pressions et menaces de toute sorte (re-)devenaient une activité hautement rentable. La mécanique infernale s'est alors enclenchée : en janvier 2008, les partenaires sociaux ont soit disant « modernisé » le marché du travail en permettant le départ en préretraite à 57 ans ; en avril 2008 la CGT et la CFDT ont réussi à accroître leur pouvoir aux dépens des autres syndicats sans que l'accord final ne règle la question de l'opacité du financement des organisations syndicales ; en mai les taxis ont considérablement amélioré leur situation aux dépens des usagers ; en août les principales enseignes de la grande distribution ont consolidé leurs position de monopole; toujours en août la détaxation des heures supplémentaires, dont le dispositif rappelle par son absurdité celui de l'impôt sur les portes et fenêtres institué par le directoire en 1798, aboutit à plusieurs milliards d'euro de rentrées fiscales en moins chaque année sans aucun effet avéré sur l'activité ; enfin, en décembre 2008, les 18 premiers mois du quinquennat s'achèvent avec le rSa, une peau de chagrin qui contribue à opacifier la carte des minima sociaux sans accroître notablement les revenus des moins bien lotis.

Nicolas Sarkozy a sous estimé les intérêts et les capacités de ses interlocuteurs. L’hyper volontarisme, fût-il celui d'un hyper président, ne peut convaincre des individus d'agir pour le bien collectif à l'encontre de leurs propres intérêts. Il ne peut y avoir de réforme réussie dans le domaine économique sans avoir aussi une vision claire des objectifs et des moyens à utiliser pour les atteindre ${ }^{17}$. On peut légitimement douter de l'existence d'une telle vision. Pourtant, les enseignements des réformes menées dans toutes les régions du monde sont sans ambiguïté : nous devons prioritairement améliorer la qualité de notre démocratie sociale et de notre démocratie politique.

\section{Une démocratie sociale en panne}

\footnotetext{
${ }^{17}$ Voir l'article de Thierry de Montbrial , « Réforme, déclin, révolution » dans Thierry de Montbrial (éd.), Réformes-
} révolutions, le cas de la France, PUF, 2003, pp. 1-26. 
En premier lieu, nous devons modifier en profondeur le rôle et la représentativité des syndicats. Seuls $8 \%$ des salariés français sont syndiqués, principalement dans le secteur public. Les syndicats survivent grâce aux positions qu'ils occupent dans la gestion de certains services collectifs comme l'assurance chômage, la médecine du travail, les caisses de retraite ou encore les organismes collecteurs de la formation professionnelle. Ils survivent aussi grâce aux décharges syndicales et aux mises à disposition que leur octroient quelques grandes administrations ou entreprises du secteur public. Le contribuable et l'usager financent ainsi de manière opaque, et souvent à la limite de la légalité, l'équivalent d'au moins 40000 postes à temps plein mis à disposition des syndicats ${ }^{18}$. Pour les organisations syndicales, tout changement est d'abord apprécié à l'aune de ses conséquences sur l'appareil et non à celle des besoins du monde du travail. Dans ces conditions, la stratégie du gouvernement demandant aux partenaires sociaux de s'auto réformer, en leur proposant de transformer leurs accords en loi, ne pouvait aboutir qu'à des réformes en trompe l'oeil renforçant les positions de quelques-uns au détriment du plus grand nombre.

L'histoire récente des pays du nord de l'Europe ou de l'Allemagne montre pourtant que les syndicats peuvent avoir un rôle majeur à jouer dans le processus de réforme. Pour cela, leur présence dans les entreprises privées, doit être massive. Contrairement à une idée reçue, une forte présence syndicale dans l'entreprise est un gage de bonnes relations entre les employeurs et les employés. Ainsi, le Forum Economique Mondial, plus connu sous le nom de Forum de Davos et peu suspect de sympathie envers les syndicats, mène tous les ans une enquête auprès de milliers de managers dans le monde entier en leur posant la question «Les relations entre employées et employeurs sont elles conflictuelles ou coopératives ?». Sur 134 pays la France se classe $132^{\text {ième }}$, il n’y a que le Népal et le Venezuela où les relations de travail sont plus conflictuelles ${ }^{19}$ ! Et cela ne date pas d'hier. Nous occupons la queue du peloton au moins depuis 1993. Les relations sociales dans l'entreprise française sont exécrables et depuis longtemps. A l'inverse, le Danemark avec $75 \%$ de salariés syndiqués occupe la première place de ce classement. Plus généralement, tous les pays industrialisés dont le taux de syndicalisation est élevé affichent une forte coopération dans les relations de travail.

La réforme du rôle et de la représentativité des syndicats est prioritaire. Il est illusoire de penser que l'on pourra résoudre le problème des retraites, améliorer le fonctionnement de la

18 Voir Dominique Andolfatto, «Les mises à disposition : une subvention cachée pour les syndicats », 2007, disponible sur le site www.ifrap.org/HorsSC/entretien-dominique-andolfatto.htm\#1.

${ }^{19}$ Voir le tableau 7.01 intitulé « cooperation in labor-employer relations », p. 432, du Global Competitiveness Report 2008-2009, World Economic Forum. 
formation professionnelle et la gestion de l'assurance chômage si les syndicats ont toujours aussi peu d'adhérents avec une pléthore de permanents payés par le contribuable et si leur financement reste toujours aussi opaque. Pire, tenter de mener des réformes dans ces domaines sans s'attaquer aux dysfonctionnements du paritarisme risque fort d'amplifier les problèmes que l'on cherche à résoudre, comme le montrent nombre d'épisodes de réformes ratées décrites dans ce livre. Les syndicats ne peuvent devenir un moteur de la réforme que s'ils sont essentiellement présents dans l'entreprise et moins ailleurs. Cette orientation suppose la mise en place d'un «syndicalisme de service » où les adhérents payent leur cotisation pour bénéficier de prestations qui leurs sont réservées. Les intérêts de l'appareil syndical se confondent alors avec ceux du monde du travail. C'est ainsi que les pays du nord de l'Europe ont pu construire un syndicalisme volontiers réformateur sans dommage pour l'efficacité économique, bien au contraire.

\section{Les dysfonctionnements de la démocratie politique}

L'autre priorité touche au fonctionnement de notre démocratie politique. L'Assemblée nationale et le Sénat ont plus tendance à opacifier les textes et à les vider de leur contenu qu'à les améliorer. Tel est le cas pour la loi de modernisation du marché du travail, dont l'effet potentiellement négatif sur l'emploi des seniors résulte d'amendements législatifs. Tel est aussi le cas de la loi de modernisation de l'économie, où les défauts de la législation sur la grande distribution, supprimés en grande partie par le projet de loi déposé par le gouvernement, ont été réintroduits par divers amendements votés par les députés et les sénateurs. Lorsqu'ils auraient pu améliorer substantiellement les projets de loi, députés et sénateurs n'en ont pas saisi l'opportunité. Ainsi, le projet de loi sur la modernisation du dialogue social contenait plusieurs articles qui ne permettaient pas, à l'évidence, d'atteindre les objectifs annoncés de transparence financière des syndicats. Rien d'étonnant à cela, puisque le projet de loi était la transcription de la «position commune» adoptée par une partie des partenaires sociaux qui préféraient ne pas trop s'engager sur le sujet. Au lieu de prendre le problème à bras le corps, députés et sénateurs se sont contentés de voter un texte flou, qui ne change pratiquement rien en matière de transparence financière des organisations syndicales.

En réalité, les parlementaires, principalement motivés par la défense d'intérêts locaux, sont souvent dépassés par la complexité des dossiers sur lesquels ils doivent délibérer. Ils sont influencés par des lobbies de toutes sortes qui leur «inspirent» bon nombre d'amendements ${ }^{20}$. Ces derniers sont déposés par des parlementaires «bien informés», souvent correspondants

\footnotetext{
${ }^{20}$ Voir l'enquête de Hélène Constanty et Vincent Nouzille, dans leur ouvrage Députés sous influences, le vrai pouvoir des lobbies à l'Assemblée nationale, Fayard, 2006.
} 
officieux des lobbies, et qui disposent d'un pouvoir de persuasion d'autant plus important que les autres parlementaires maitrisent mal les dossiers. Ces dysfonctionnements du parlement ne sont pas une fatalité. Ils sont la conséquence de défauts mainte fois dénoncés qu'il est possible de changer. Le Comité «sur la réforme et le rééquilibrage des institutions » présidé par Edouard Balladur et chargé de préparer la réforme constitutionnelle qui fut finalement votée par le Congrès réuni à Versailles le 21 juillet 2008, soulignait que le «réequilibrage des institutions passe d'abord, dans le cadre du régime tel qu'il fonctionne aujourd'hui, par un accroissement des attributions et du rôle $d u$ Parlementr $^{21}$. Il a exprimé de nombreuses propositions pour atteindre cet objectif.

A ce titre, il a émis un jugement sans appel sur le cumul des mandats : "L'activité parlementaire de législation et de contrôle constitue, par elle même, une activité à temps plein. Aussi le Comité est-il d'avis que l'interdiction du cumul des mandats et des fonctions est la seule mesure qui corresponde vraiment aux exigences d'une démocratie parlementaire moderne ». Pour le Comité Balladur, l'interdiction du cumul des mandats n'est pas une mesure parmi d'autres. C'est «la seule mesure qui corresponde vraiment aux exigences d'une démocratie parlementaire moderne ». Guy Carcassonne, spécialiste de droit constitutionnel, va encore plus loin. Pour lui «la suppression du cumul des mandats pour les députés n'est pas une réforme parmi les autres. En l'état de nos institutions, elle est la mère de toutes les autres ${ }^{22}$.

A l'heure actuelle, parmi les 577 députés, pratiquement tous sont au moins conseillers municipaux ou généraux, 259 sont maires, 21 sont présidents de conseil général, 8 sont présidents de conseil régional ; sur les 331 sénateurs, 121 sont maires, 32 sont présidents de conseil général et 3 sont présidents de conseil régional. Cette situation de cumul d'un mandat national et local est unique parmi les grandes démocraties occidentales ${ }^{23}$. En guise de conseil aux nouveaux députés, on prête à Valérie Giscard d'Estaing les propos suivants : «ceux que l'on voit beaucoup à l'Assemblée, on ne les voit pas longtemps ». En effet, pour avoir des chances d'être réélu député ou sénateur, il faut être surtout être présent dans sa circonscription. Il en résulte que nos représentants élus à l'Assemblée nationale et au Sénat n'y passent, pour la plupart d'entre eux, que peu de temps. En outre, ils y ont, à cause de leur grand nombre, des moyens limités. Ils disposent chacun d'un ou

\footnotetext{
${ }^{21}$ Edouard Balladur, Une cinquième République plus démocratique - Comité de réflexion et de proposition sur la modernisation et le rééquilibrage des institutions de la cinquième République, La documentation française, 2007, page 4.

22 «Comment mettre fin au cumul des mandats», Libération, 17 janvier 2007. De nombreux observateurs de la vie politique partagent cette opinion. C'est le cas en particulier de Jean-Michel Apathie qui dénonce régulièrement sur son blog les méfaits du cumul des mandats. Voir par exemple son billet du 11 décembre 2008 à l'adresse http://blogs.rtl.fr/aphatie/index.php/?q=cumul+des+mandats.

${ }^{23}$ Voir les études de Michaël Cheylan et Philippe Manière, «Député, un job à temps plein », Briefing paper, Institut Montaigne, décembre 2006, disponible à www.institutmontaigne.org, et de Yves Mény, "Des mœurs irréformables ? », Pouvoirs, n 126, pp. 37-45, 2008. Outre le cumul des mandats et la faiblesse du Parlement, Yves Mény souligne d'autres singularités françaises qui ne contribuent pas à la qualité de notre démocratie politique : la présence très minoritaire des femmes dans les instances élues et la faible portée juridique et pratique des textes votés (nous aurions beaucoup de « lois bavardes »).
} 
deux assistants parlementaires ${ }^{24}$, ce qui ne leur permet pas d'avoir accès à une capacité d'expertise suffisante pour suivre l'ensemble des dossiers ${ }^{25}$. Nous avons vu, par exemple, que lors du vote sur un article crucial de la loi de modernisation du marché du travail, il devait y avoir une quarantaine de députés en séance et il n'était pas sûr qu'ils saisissaient tous parfaitement les conséquences du texte qu'ils étaient en train de voter. Cette faiblesse des moyens dont disposent les députés et sénateurs est une contrepartie de leur nombre pléthorique. La comparaison de la France avec les autres pays développés montre en effet que nous avons un nombre très élevé de représentants élus par habitant au niveau national ${ }^{26}$.

Nous avons fait le choix d'avoir un grand nombre de parlementaires, exprimant avant tout des intérêts locaux avec des moyens trop limités pour se saisir vraiment des problèmes qui concernent l'ensemble des Français. Les conséquences en sont désastreuses et bien connues ${ }^{27}$ : une désorganisation du travail parlementaire concentré sur deux jours par semaine, un biais très important pour les intérêts locaux au détriment de l'intérêt général ce qui les rend plus « compréhensifs » aux demandes émanant de tous les groupes de pression ; une faible capacité à contrer les actions de quelques députés «informés » par des lobbies qui les ont ciblés. Cette situation a aussi des effets désastreux sur la perception des parlementaires et du fonctionnement de l'ensemble des institutions : un quart des Français déclarent ne faire «absolument pas confiance » au parlement, alors que cette proportion est inférieure à $5 \%$ dans les pays scandinaves $^{28}$.

La recommandation de la fin du cumul des mandats par le Comité présidé par Edouard Balladur n'est donc pas une surprise. Elle était en fait attendue. Pourtant, le projet de loi constitutionnelle déposé à l'Assemblée nationale ne reprendra pas cette proposition. Les parlementaires de la majorité, et ils étaient loin d'être les seuls, ont exercé un véritable chantage en menaçant de ne pas voter le projet auquel Nicolas Sarkozy tenait particulièrement, si toute référence au cumul des mandats n'était pas écartée. A l'instar de bon nombre de projets de

\footnotetext{
${ }^{24}$ Un député peut embaucher jusqu'à 5 collaborateurs mais il ne dispose que d'un crédit mensuel de 8949 euros pour les rémunérer.

${ }^{25}$ On trouvera une description intéressante de la vie quotidienne d'un député dans l'ouvrage de Laurent Wauquiez, Un huron à l'assemblée nationale, petit manuel à l'attention de ceux qui veulent secouer la politique, Editions Privé, 2006.

${ }^{26}$ Voir Emmanuelle Auriol et Robert Gary-Bobo, « On the Optimal Number of Representatives », IDEI Working Paper, n86, mars 2008.

27 Voir, «Député, un job à temps plein », Briefing Paper, Institut Montaigne, décembre 2006. Disponible à www.institutmontaigne.org et Guy Carcassonne, "Qui va à la chasse doit perdre sa place », dans Telos, Les vingt chantiers de l'Elysée, Propositions pour 2007, pp. 13-23, Hachette littérature, 2007.

${ }^{28}$ Voir Yann Algan et Pierre Cahuc, 2007, La société de défiance : comment le modèle social français s'auto-détruit? Edition rue d'Ulm.
} 
réformes des 18 premiers mois de son quinquennat, Nicolas Sarkozy a cédé sur cette mesure essentielle $^{29}$, nécessaire à la mise en œuvre d'un véritable renforcement du rôle du parlement.

Toute réforme dans le domaine économique et social - et probablement ailleurs - aura toujours aussi peu de chances de succès sans, qu'au préalable, des modifications importantes de notre démocratie sociale et de notre démocratie politique soient mises en oeuvre. La réforme de la démocratie sociale voudrait que l'on s'engage vers un syndicalisme de service permettant d'assurer la force et l'indépendance financière des organisations syndicales principalement grâce au nombre de leurs adhérents. La réforme de la démocratie politique suppose que l'on renforce le parlement, en particulier en mettant fin au cumul des mandats et en améliorant les moyens dont disposent nos parlementaires. L'influence des groupes de pression pour faire obstacle au changement ou en tirer tous les bénéfices aurait alors plus de chance d'être mieux maittrisée. La qualité des textes de loi et l'évaluation des politiques publiques mises en œuvre par l'exécutif ne pourraient aussi être qu'améliorées. Faute d'avoir fait un préalable de ces deux chantiers et de les avoir menés à terme, il y avait peu de chances de réussir des changements d'envergure. C'est là, principalement, que résident les vraies raisons de l'échec des réformes entreprises par le président Sarkozy au cours des deux premières années de son quinquennat ${ }^{30}$.

\footnotetext{
${ }^{29}$ Le comité Balladur préconise aussi d'interdire tout cumul entre une fonction ministérielle et un mandat électif. Cette recommandation est aussi absente de la réforme constitutionnelle.

${ }^{30}$ Une analyse détaillée de l'échec des réformes économiques et sociales se trouve dans notre ouvrage, Les Réformes ratées du président Sarkozy, Flammarion, mars 2009.
} 